\section{PHASE 1 STUDY IN HEALTHY VOLUNTEERS TO ASSESS PK, PD SAFETY \& TOLERABILITY OF BOS161721, AN EXTENDED HALF-LIFE ANTI-IL21 MONOCLONAL ANTIBODY}

${ }^{1}$ Xavier Valencia*, ${ }^{1}$ Christen Glogowski, ${ }^{2}$ Azra Hussaini, ${ }^{1}$ Richard Mountfield, ${ }^{1}$ Peter Ho. ${ }^{1}$ Boston Pharmaceuticals; ${ }^{2}$ Parexel Clinical Research Unit

10.1136/lupus-2019-Ism.24

Background Interleukin-21 (IL-21) is a pleiotropic cytokine. Both IL-21 and the IL-21 receptor (IL-21R) have been shown to be upregulated in systemic lupus erythematosus (SLE). BOS161721, a humanized monoclonal immunoglobulin (IgG1) that binds to and neutralizes IL-21. It is currently in development for the treatment of SLE. The primary objective of this trial was to assess safety and tolerability of single intravenous (i.v.) and subcutaneous (s.c.) doses of BOS161721 in healthy volunteers (HV). Methods A phase 1, randomized, single-center, placebo-controlled, double-blind, single-dose-escalation trial was conducted in male and female HV $(n=61)$ aged $18-55$ years. Subjects were randomized in a 3:1 ratio (BOS161721: placebo) and received either a single SC dose of BOS161721 (1\#, 3, 10, $22 \#, 30,60,120$, or $240 \mathrm{mg}$, \#=i .v. administration) or placebo. Key safety parameters included adverse events (AEs), injection-site reactions and detection of neutralizing antibodies (nAb) against BOS161721. Pharmacokinetic (PK) and pharmacodynamic (PD) parameters included pStat3 levels and expression levels of IL-21 gene signature in blood.

Results A total of 39 treatment emergent AEs were reported in 47 subjects $(83 \%)$. The most commonly reported related AE was influenza-like illness (6.4\%). No dose dependency was detected for AEs. One serious AE (fatal pulmonary embolism) was reported 127 days post dosing in a subject exposed to $240 \mathrm{mg}$ SC of BOS161721; it was evaluated as not related to the study drug by the investigator. No nAbs were detected. No clinically relevant changes in laboratory parameters, vital signs or ECG were observed. Dose-linearity PK was observed with SC administration and Tmax occurred at approximately 6 days posts dose, indicating no evidence of target mediated clearance. The mean terminal elimination half-life of BOS161721 was estimated to be 80 days at the highest dose of $240 \mathrm{mg}$. Notably, a dose dependent decrease was observed for the pSTAT3 parameter indicating complete neutralization of IL-21 signaling at doses of $60 \mathrm{mg}$ and greater.

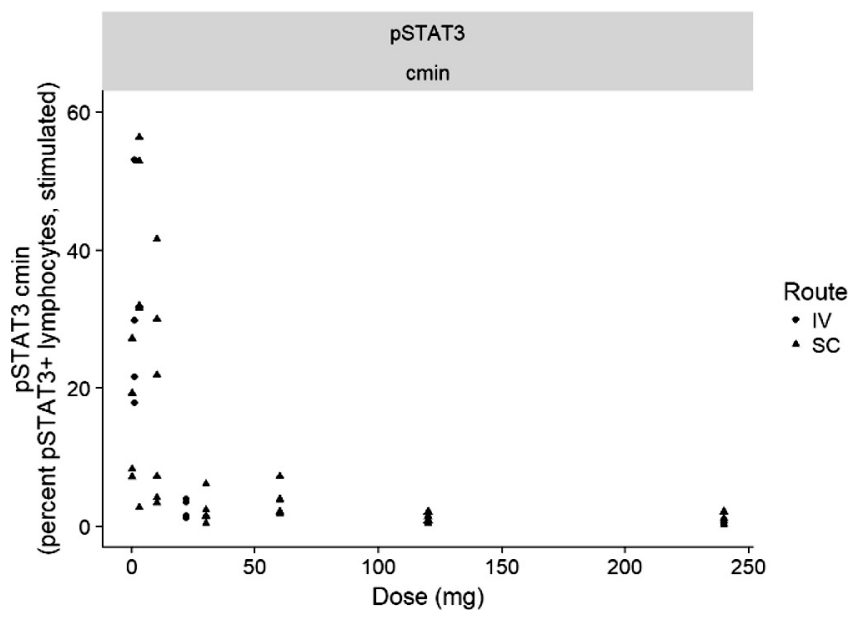

Abstract 24 Figure 1 pSTAT3 figure
Conclusions BOS161721 was safe and well tolerated in HV. Dose-linear PK of BOS161721 was demonstrated in HV. Importantly, BOS161721 potently suppressed IL-21 induced signaling which demonstrates the expected biologic and potential clinical activity of BOS161721. A phase $1 \mathrm{~b}$ study is currently ongoing in patients with SLE.

Funding Source(s): Boston Pharmaceuticals

\section{PROSPECTIVE MULTICENTER VALIDATION OF THE LUPUS LOW DISEASE ACTIVITY STATE (LLDAS) TREATMENT TARGET}

${ }^{1}$ Eric Morand*, ${ }^{2}$ Vera Golder, ${ }^{2}$ Rangi Kandane-Rathnayake, ${ }^{3}$ Molla Huq, ${ }^{2}$ Hieu Nim, ${ }^{4}$ Worawit Louthrenoo, ${ }^{5}$ Shue Fen Luo, ${ }^{6}$ Aisha Lateef, ${ }^{7}$ Sargunan Sockalingam, ${ }^{8}$ Sandra Navarra, ${ }^{9}$ Laniyati Hamijoyo, ${ }^{10}$ Yasuhiro Katsumata, ${ }^{11}$ Madelynn Chan, ${ }^{2}$ Alberta Y Hoi, ${ }^{3}$ Mandana Nikpour. ${ }^{1}$ Monash University, Melbourne, Australia; ${ }^{2}$ Monash University; ${ }^{3}$ Melbourne University; ${ }^{4}$ Chiang Mai University Hospital; ${ }^{5}$ Chang Gung Memorial Hospital; ${ }^{6}$ National University Hospital; ${ }^{7}$ University of Malaya; ${ }^{8}$ Santo Tomas Hospital; ${ }^{9}$ Padjadjaran University; ${ }^{10}$ Tokyo Women's University; ${ }^{11}$ Tan Tock Seng Hospital

\subsection{6/lupus-2019-Ism.25}

Background Adoption of treat to target approaches for Systemic Lupus Erythematosus (SLE) requires the definition of a target state validated for improved patient outcomes. The Lupus Low Disease Activity State (LLDAS) has been shown in multiple retrospective and cross-sectional studies to have face, content, construct and criterion validity and be associated with better quality of life. We report on a multinational prospective study undertaken to determine whether LLDAS attainment is associated with protection from flare and damage accrual.

Methods A prospective multinational cohort study was undertaken in 13 centres between 2013-2017. Patients with SLE were recruited, SLEDAI-2k, SELENA flare index, PGA, and medication data collected at every visit, and damage (SLICCACR damage index (SDI)) collected annually. Time-dependent Cox proportional hazards models were used to assess the association of LLDAS at any time point, as well as the effect of the proportion of time spent in LLDAS, with disease flare and damage accrual (increase in SDI).

Results 1735 patients were followed for (mean \pm SD) 2.2 \pm 0.9 years, totalling 12717 visits. LLDAS was achieved in $54.6 \%$ of visits. Attainment of LLDAS at any timepoint was associated with reduction in subsequent flare (HR 0.65 , 95\% CI $0.56-0.76, \mathrm{p}<0.001$ ) and damage accrual (HR 0.55, 95\% CI $0.43-0.70, \mathrm{p}<0.001)$. Similarly, patients who spent $50 \%$ of their observed time in LLDAS had reduction in risk of flare (RR 0.41, p<0.001) and damage accrual (RR 0.59, $\mathrm{p}<0.001$ ), compared to those with $<50 \%$ of observed time in LLDAS. Increased durations of sustained LLDAS were associated with incremental reduction in risk of damage accrual. The protective association of LLDAS with reduced damage accrual was maintained in subgroup analysis of patients with active disease at baseline (SLEDAI-2K>6) or pre-existing damage (SDI $>0)$.

Conclusions LLDAS attainment provides significant protection against disease flare and damage accrual thereby supporting its use as a treatment target in SLE, and as an outcome measure for clinical trials and treat-to-target strategies.

Funding Source(s): The Asia Pacific Lupus Collaboration receives project support grants from UCB, GlaxoSmithKline, Janssen, Bristo-Myers Squibb, and AstraZeneca. 